\title{
Men versus Women
}

\section{Does Size Matter in Total Knee Arthroplasty?}

\author{
Roger H. Emerson Jr MD, Jessica Martinez MD
}

Published online: 16 August 2008

(c) The Association of Bone and Joint Surgeons 2008

\begin{abstract}
The role played by femoral component sizing in the clinical outcome of primary TKA is currently debated. Oversizing the femur in patients with smaller knees could lead to overstuffing the knee capsule with resulting pain and reduced range of motion. We asked whether the distribution of femoral component sizes differed between genders and whether the availability of additional sizes benefited genders differently and led to a measurable improvement in knee flexion and Knee Society scores or pain. We retrospectively examined two groups of consecutive knees of patients who underwent primary TKA using similar techniques and constraint: Group 1 (93 men and 90 women) who had available four original sizes and Group 2 (106 men and 106 women) after the introduction of three new smaller sizes. More than twice as many new smaller sizes were used in women $(52.3 \%, 56$ of 106) compared to men $(17.9 \%, 19$ of 106). At the scheduled 6-month followup visit (average, 6 months; range, 5-7 months), we found no differences in the knee score, pain score, and knee flexion in men and women before the additional sizes (Group 1) and after the new sizes (Group 2). Additional sizes therefore did not appear to influence shortterm outcomes.
\end{abstract}

One of the authors (RHE) receives royalties from Biomet Inc (Warsaw, IN)

Each author certifies that his or her institution has approved the human protocol for this investigation, that all investigations were conducted in conformity with ethical principles of research, and that informed consent for participation in the study was obtained.

R. H. Emerson Jr ( $₫)$, J. Martinez

Texas Center for Joint Replacement, Presbyterian Hospital of Plano, 5940 W. Parker, Suite 100, Plano, TX 75093, USA

e-mail: rhemerson@msn.com
Level of Evidence: Level III, therapeutic study. See the Guidelines for Authors for a complete description of levels of evidence.

\section{Introduction}

Contemporary total knee implant designs provide a range of sizes to achieve the best fit in nearly all patients. Sizing recommendations for TKA seek to maximize the size of the tibial component and minimize the size of the femoral component. The rationale for these recommendations is to have the tibial tray large enough for bony support from the tibial cortex and the femoral component small enough to avoid overexpanding the soft tissue capsular envelope of the knee and extensor mechanism (but without notching the anterior femoral cortex), thereby reducing knee range of motion and potentially causing pain.

A recent claim has been made that, with current size offerings, missizing, especially for the femur, resulting in excessive mediolateral (ML) overhang, may occur more commonly than previously thought and more frequently in women than in men [1]. Anatomic studies demonstrate differences in the shape of the distal femur between men and women. For any given anteroposterior (AP) femoral dimension, women tend to have a narrower ML dimension than men [2]. Hitt et al. [6] compared the AP/ML ratio (aspect ratio) in 209 female and 128 male knees to the dimensions of six contemporary prosthetic knee systems. For women, they found an association between the component size and the amount of ML overhang, with larger sizes having more overhang. The implication is that past size offerings have been too large on average for women and that this increase would influence the outcomes by over-tightening the knee capsule causing pain and reduced range of motion. Until recently 
concern about such femoral size mismatch has not been raised in the TKA literature, suggesting it was not perceived as a clinically important problem.

Do women benefit, therefore, more than men if smaller sizes are made available? Does the use of more sizeappropriate femoral implants lead to any measurable clinical benefit? Specifically, does it result in better range of motion or more comfort in the knee? We hypothesized traditional femoral sizing is appropriate and we would find no difference in knee flexion or pain or knee function for men and women after the addition of more sizes to an otherwise contemporary TKA design.

\section{Materials and Methods}

This null hypothesis was tested by taking advantage of the rollout of new sizes for a contemporary total knee system (Vanguard $^{\mathrm{TM}}$ Total Knee System; Biomet Inc, Warsaw, IN). In 2003, the Vanguard ${ }^{\mathrm{TM}}$ Total Knee System was introduced with four available sizes, $60,65,70,75$, referring to the ML dimension of the femur. The aspect ratio (AP/ML dimension) was 0.82 (data from Biomet Inc., Warsaw, IN). These same sizes had historically been used in preceding designs since 1983, with clinical experience confirming good function and survivorship [3,11]. In July 2004, three additional femoral sizes were made available with the same aspect ratio, which expanded the size offerings to seven; these "half sizes" included the new sizes $57.5,62.5$, and 67.5.

From an overall series of 620 consecutive patients who underwent primary TKA performed by the senior author (RHE) using the Vanguard ${ }^{\mathrm{TM}}$ Total Knee System, a study series was retrospectively created, consisting of 393 patients who underwent primary TKA with a cruciatepreserving knee design, including patients with osteoarthritis, rheumatoid arthritis, and avascular necrosis. We excluded patients with severe posttraumatic arthritis with prior intraarticular fracture or requiring a cruciate-substituting design. We then created two cohorts, one cohort using the original available sizes (Group 1) and another using the expanded set of sizes introduced 1 year later (Group 2). Group 1 (183 patients) consisted of 93 men and 90 women, and Group 2 (212 patients), selected from those patients immediately after availability of the new sizes, consisted of 106 men and 106 women. The two groups were comparable in terms of age, gender, and diagnosis (Fig. 1). In Group 1, the average age of patients was 63.4 years in men and 65.8 years in women. In Group 2, the average age of patients was 63.8 years in men and 66.3 years in women. The preoperative knee flexion was not different in men and women between the two groups (women: $109.6^{\circ}$ in Group 1 and $114^{\circ}$ in Group 2

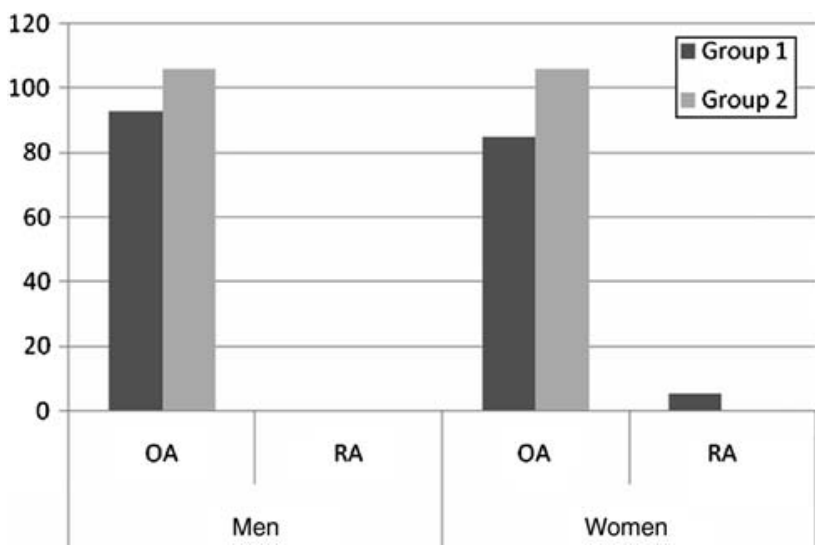

Fig. 1 A graph shows the distribution of diagnoses between Groups 1 and 2. The diagnoses are similar between the two study groups. $\mathrm{RA}=$ rheumatoid arthritis; $\mathrm{OA}=$ osteoarthritis.

[p $=0.019$ ]; men: $112.9^{\circ}$ in Group 1 and $114.3^{\circ}$ in Group $2[\mathrm{p}=0.39])$. The IRB approved the study design.

Assuming an alpha error of 0.05 (universally accepted) and a beta error of 0.2 (upper limit of accepted) and by assuming a "power index" of 2.80 (giving an $80 \%$ power of detecting a difference) with a standard deviation of $11^{\circ}$ (measured from the data), it would take a series of 210 patients (105 in each group) to show a $6^{\circ}$ difference in the mean ranges of motion between the two independent groups [5]. Therefore, the size of the series is sufficient to avoid a Type 1, alpha, error, falsely rejecting the null hypothesis, in this case, that femoral sizing does not make a difference in range of motion. The series would have to be much larger to avoid a Type 2 error, falsely supporting the null hypothesis when in fact there is a genuine difference between Groups 1 and 2.

The anesthesia choices and surgical techniques were similar throughout the study timeframe. Patients had a regional anesthetic unless contraindicated by a medical issue. All implants were fully cemented posterior cruciate ligament-retaining with a modular 2-mm posterior-lipped articulating surface. The components were placed with instrumentation supplied by the manufacturer, using intramedullary guides on the femur and extramedullary guides on the tibia. Posterior referencing was used to orient the femoral component, choosing the smallest that would fit anteroposteriorly without notching the anterior cortex of the femur. Such referencing maintains a constant flexion gap, unrelated to femoral size, required when preserving the posterior cruciate ligament, and only increases the ML and anterior dimension as the femoral size increases. Femoral rotation was determined by the transverse epicondylar axis. The femoral component was routinely placed as laterally as possible on the distal femur, without overhang, to promote best patellar tracking. When in between sizes, the larger size was used, without regard to ML 
coverage, to avoid notching. No specific notation of overhang was made. Soft tissue balancing was the same for each group. Posterior cruciate ligament releasing was commonly used to expand the flexion gap if rollback was excessive. Both groups had the same postoperative management with inpatient and outpatient physical therapy. Followup was performed at scheduled visits, at 1 and 2 months by a physical therapist and at 6 months by the operating surgeon, and then ongoing implant monitoring was recommended every 2 years.

The senior author (RHE) evaluated all patients at 6 months using the Knee Society score [4] and passive range of motion measured using a standard long goniometer with the knee in the supine position and the center of the knee aligned with the greater trochanter and lateral malleolus. The data were collected without knowledge of the implant size.

The range of motion and clinical score data were assumed to follow a normal distribution, with unequal variance, and with appropriate means, allowing for the $t$ test using two tails. Effect size was determined by Cohen's $\mathrm{d}$ designed to be used in the context of a t test on means, where $d=0.2$ is indicative of a small effect, $d=0.5$ a medium effect, and $d=0.8$ a large effect size. The chi square test was used to compare sizes used by men and women.

\section{Results}

Do women benefit more than men if additional and smaller sizes are made available? As expected, we used more of the new smaller sizes in women than men. In Group 1, we used more $(\mathrm{p}<0.001)$ Size 65 and below in women than in men (76 of 90 or $84.4 \%$ versus nine of 93 or $9.6 \%$ respectively) in men, and more $(\mathrm{p}<0.001)$ Size 70 and above in men than in women (versus 84 of $9390.3 \%$ versus 14 of 90 or $15.5 \%$, respectively) (Fig. 2). In Group 2, we also used more $(\mathrm{p}<0.01)$ Size 65 and below in women than in men (83 of 106 or $78.3 \%$ versus 10 of 106 or $9.3 \%$, respectively), and more $(\mathrm{p}<0.001)$ Size 70 and above in men than in women (96 of 106 or $90.4 \%$ versus 23 of 106 or $21.6 \%$, respectively) (Fig. 3).

The relative distribution of the new sizes differed by gender, with women benefiting more than men from the availability of new smaller sizes: women used $52.3 \%$ (56 of 106 ) of the new sizes and $47.3 \%$ (44 of 106) of the original sizes whereas men used only $17.9 \%$ (19 of 106) of the new sizes and $82.1 \%$ (81 of 106) of the original sizes.

Does the use of more size appropriate femoral implants lead to any measurable clinical benefit? Specifically, does it result in better range of motion or more comfort in the knee? The postoperative clinical scores at 6 months for

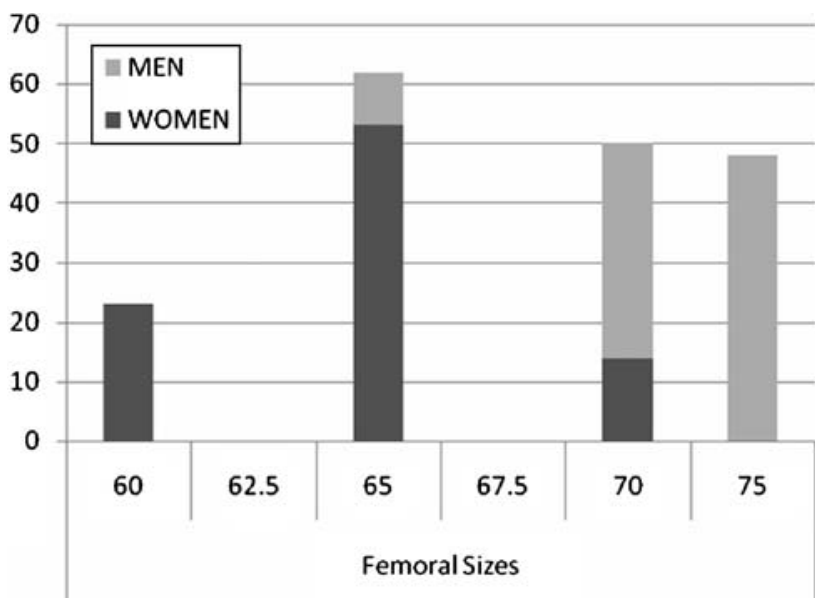

Fig. 2 A graph shows the distribution of femoral sizes for men and women in Group 1. Size 65 is the most common size used. Women use more small sizes than men.

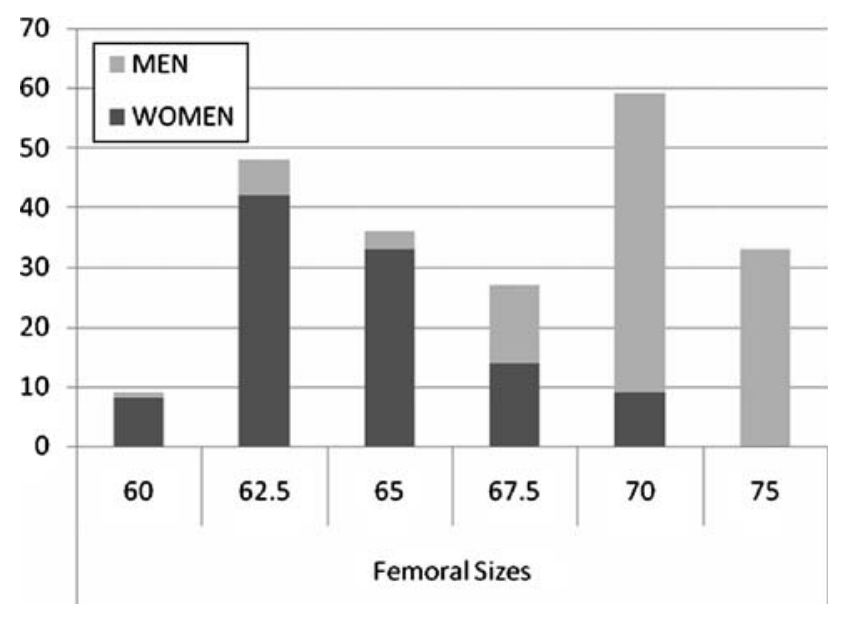

Fig. 3 A graph shows the distribution of femoral sizes for men and women after the introduction of half sizes in Group 2. Size 62.5 is the most common size used. Women benefited more than men from the addition of newer half sizes. Note some women had an increase in femoral component size from Size 60 and others had a decrease in femoral component size from Size 65.

Group 1 were 93.3 (range, 65-100) for women and 94.7 (range, 70-100) for men (Fig. 4) and the postoperative clinical scores for Group 2 were 93.3 (range, 60-100) for women and 92.8 (range, 70-100) for men (Fig. 5).

Postoperative flexion for both men and women was similar before and after the introduction of the additional sizes. In Group 1, mean postoperative flexion was similar $(\mathrm{p}=0.11)$ for men (mean, $116.2^{\circ}$; range, $\left.50^{\circ}-133^{\circ}\right)$ and women (mean, $114.0^{\circ}$; range, $85^{\circ}-132^{\circ}$ ). In Group 2, the mean postoperative flexion was also similar for men (mean, $113.8^{\circ}$; range, $84^{\circ}-130^{\circ}$ ) and women (mean, $115.8^{\circ}$; range, $77^{\circ}-135^{\circ}$ ) (Fig. 6). Among women mean knee flexion was similar $(\mathrm{p}=0.29)$ in Group 1 and Group 2$)\left(114.0^{\circ}\right.$ vs $115.8^{\circ}$, respectively). 


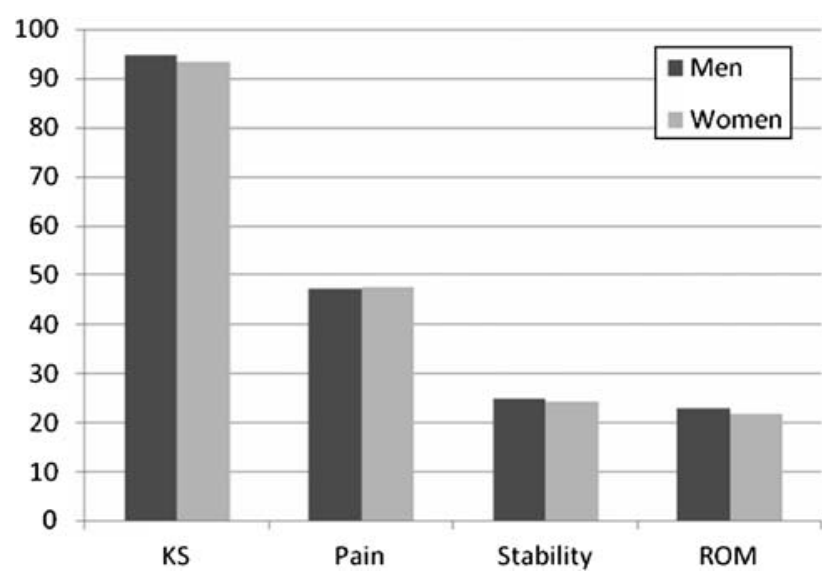

Fig. 4 A graph showing postoperative clinical scores in Group 1 indicates no differences between men and women. $\mathrm{KS}=$ Knee Society; ROM = range of motion.

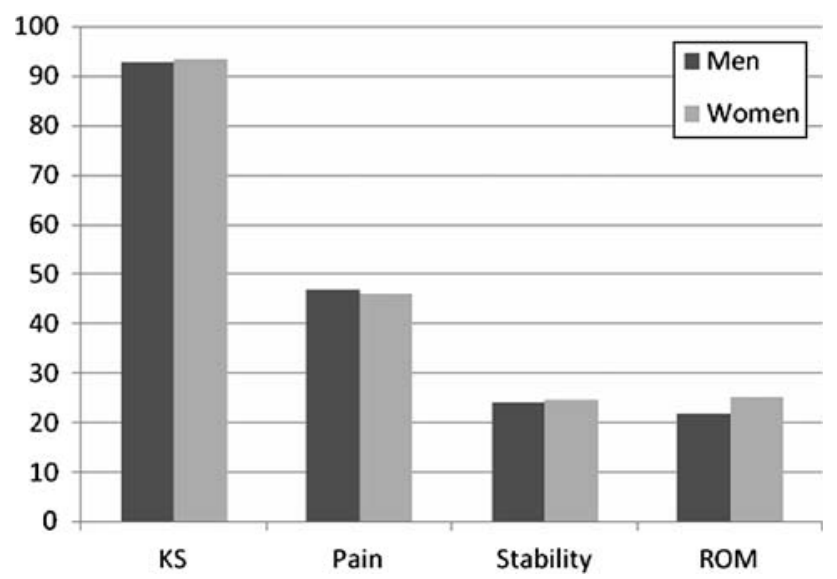

Fig. 5 A graph showing postoperative clinical scores in Group 2 indicates no differences between men and women. $\mathrm{KS}=$ Knee Society; ROM = range of motion.

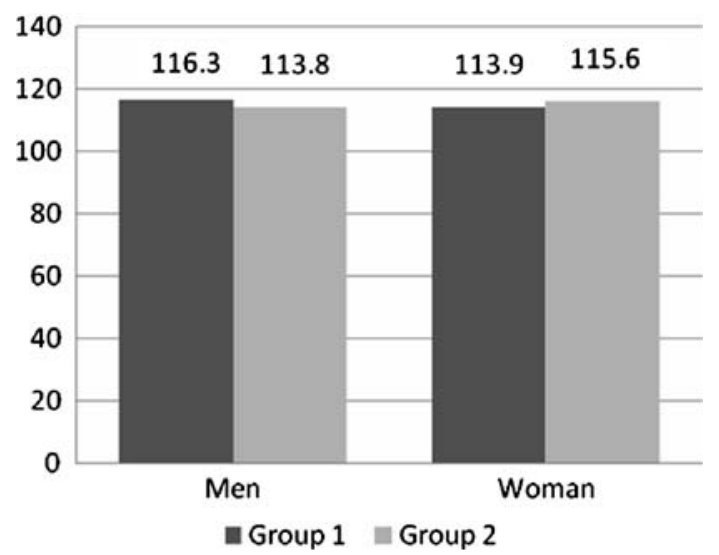

Fig. 6 A graph comparing postoperative knee flexion between Group 1 and Group 2 indicate no difference for either gender between groups. There were no differences in improvement in either group.

\section{Discussion}

It is widely believed total knee implant systems should seek to restore the knee as close as possible to normal anatomic dimensions. There are known anatomic differences in the average shape and size of the distal femur between men and women, with women using more small size implants than men $[2,6]$. Do women benefit more than men from the introduction of smaller femoral sizes to an existing range of sizes? Logically, the answer would be "yes" and this study confirms women used proportionately more of the new smaller sizes as they were made available. While the emphasis in the orthopaedic community has been on the problems of oversizing the femoral component (especially in women), there may also be an issue of undersizing. We therefore asked whether the distribution of femoral component sizes differed between genders and whether the availability of additional sizes benefited genders differently and led to a measurable improvement in knee flexion and Knee Society scores or pain.

A limitation of this study is the precision or repeatability of the Knee Society score as a means of discerning small changes in clinical outcome. Liow et al. [8] demonstrated an intraobserver difference of 11 points and interobserver difference of 16 points for the Knee Society score. Skilled observers demonstrated more consistency, however. On the other hand, knee flexion measured with a goniometer by skilled observers has more precision, with a high intraobserver correlation (0.99) and interobserver correlation (0.90) as reported by Watkins et al. [14]. Our ad hoc power analysis showed a series of 210 (105 in each group) would be sufficient to determine a $6^{\circ}$ difference in mean knee flexion. If we assume a difference in flexion truly exists, the size of the difference can be expressed as the effect size. Cohen's $d$ is the appropriate effect size measure to use in the context of a t test on means. $d$ is defined as the difference between two means divided by the pooled standard deviation for those means. In this case, $d=0.24$ for men and $\mathrm{d}=0.13$ for women, indicating a small effect, meaning it would take a very large series to discern any difference between the groups, or put another way, any difference is unlikely clinically important. Considered together, the knee flexion and clinical score results, the case supporting the null hypothesis that past femoral component sizing has been appropriate for both men and women is strong.

Our data suggest the most commonly used small size in Group 2 was Size 62.5. Therefore, some small knees in Group 2 were upsized from Size 60 and others were downsized from Size 65 . The advantage of more sizes of femur is that more knees will have a best fit, which may involve upsizing as well as downsizing. The next question was whether the use of more appropriate sizes results in a 
measurable improvement in postoperative outcomes, utilizing knee flexion and Knee Society score as measures of function and comfort? We found improvement in these parameters. The null hypothesis of this study that traditional sizing of femoral components is appropriate has been affirmed.

Our findings are consistent with the findings in the literature. Pellengahr et al. [9], in a study of 133 unconstrained TKAs, could find no association between Knee Society scores and femoral, tibial, or polyethylene insert size. Ritter et al. [12] reported no gender differences in a series of older patients with TKAs, whereas age at surgery did play a role in the surgical outcome. Kotani et al. [7], in a contemporary TKA design, reported no gender difference at 2-year followup for knee flexion, with men averaging $116.4^{\circ}$ and women $118.3^{\circ}$, in the same range as reported in our study. In addition, Kotani et al. [7] found no correlation between range of motion and femoral component size. Most long-term studies of TKA function focus on survivorship rather than knee scores and range of motion. This literature has not demonstrated consistent gender-based differences. For example, Scuderi et al. [13] reported a 15 -year survivorship of $90.5 \%$ for a series of TKAs, demonstrating no influence for gender. On the other hand, in a study of 11,606 primary TKAs, Rand et al. [10] reported better survivorship in women than in men $(93 \%$ versus $88 \%$, respectively).

Our data are for one implant design and may not apply to other contemporary designs. Going from four femoral sizes to seven femoral sizes introduces a half size between the original sizes, certainly eliminating the occasional gross mismatch that might occur with limited size availability, but $47.3 \%$ of women and $82.1 \%$ of men still used one of the original sizes. A longer followup period may yield differing results, but incremental clinical improvement beyond 6 months would likely be small and not favor one gender over another.

The best outcomes from TKA come from adherence to the established principles of restoring limb alignment, orienting components properly, balancing the soft tissues, and attaining stable implant to bone fixation. We believe small incremental changes in implant sizing, while not something to be completely ignored, should be low in the hierarchy of surgical importance. Sufficient sizes of a contemporary design should be available to prevent gross mismatch between the patient anatomy and the femoral prosthesis, but additional femoral sizes that would seemingly improve the precision of the surgery did not lead to better clinical scores or better knee flexion for either men or women with the study methods used here.

\section{References}

1. Booth R. Sex and the TKR: gender sensitive designs. Orthopedics. 2006;29:836-838.

2. Chin KR, Dalury DF, Zurakowski D, Scott RD. Intraoperative measurements of male and female distal femurs during primary total knee arthroplasty. J Knee Surg. 2002;15:213-217.

3. Emerson RH Jr, Head WC, Higgins LL. The AGC total knee prosthesis at average 11 years. J Arthroplasty. 2000;15:418-423.

4. Ewald FC. The Knee Society Total Knee Arthroplasty Roentgenographic Evaluation and Scoring System. Clin Orthop Relat Res. 1989;248:9-12.

5. Hassard TH. What sample size will I need. In: Understanding Biostatistics. Boston, MA: Mosby Year Book; 1991:175.

6. Hitt K, Shurman JR, Greene K, McCarthy J, Moskal J, Hoeman T, Mont MA. Anthropometric measurements of the human knee: correlation to the sizing of current knee arthroplasty systems. $J$ Bone Joint Surg Am. 2003;85:115-122.

7. Kotani A, Yonekura A, Bourne RB. Factors influencing range of motion after contemporary total knee arthroplasty. J Arthroplasty. 2005;20:850-856

8. Liow RY, Walker K, Wajid MA, Bedi G, Lennox CM. The reliability of the American Knee Society Score. Acta Orthop Scand. 2000;71:603-608.

9. Pellengahr C, Muller PE, Durr HR, Maier M, Birkenmaier C, Mazoochian F, Pfahler M, Troillier H, Lienemann A, Jansson V. The influence of the implant size on the outcome of unconstrained total knee arthroplasty. Acta Chir Belg. 2005;105: 508-510.

10. Rand JA, Trousdale RT, Ilstrup DM, Harmsen WS. Factors affecting the durability of primary total knee prostheses. $J$ Bone Joint Surg Am. 2003;85:259-265.

11. Ritter MA, Berend ME, Meding JB, Keating EM, Faris PM, Crites BM. Long-term followup of anatomic graduated components posterior cruciate-retaining total knee replacement. Clin Orthop Relat Res. 2001;388:51-57.

12. Ritter MA, Eizember L, Keating EM, Faris PM. The influence of age and gender on the outcome of total knee arthroplasty. Todays OR Nurse. 1995;17:10-15.

13. Scuderi GR, Insall JN, Windsor RE, Moran MC. Survivorship of cemented knee replacements. J Bone Joint Surg Br. 1989;71: 798-803.

14. Watkins MA, Riddle DL, Lamb RL, Personius WJ. Reliability of goniometric measurement and visual estimates of knee range of motion obtained in a clinical setting. Phys Ther. 1991;71: 990-996. 